\title{
Editorial
}

\section{Scope for Integration of Palliative Care in Neurological and Neurosurgical Patients}

\author{
Sachidanand J. Bharati ${ }^{1}$ Sushma Bhatanagar ${ }^{1}$ \\ 1Department of Onco-Anaesthesia and Palliative Medicine, Dr. BRA \\ Institute Rotary Cancer Hospital, All India Institute of Medical \\ Sciences (AlIMS), Ansari Nagar, New Delhi, India
}

J Neuroanaesthesiol Crit Care 2019;6:3-4

Palliative care focuses on improving the quality of life of patients with serious or advanced medical illnesses. ${ }^{1}$ Neurological and neurosurgical conditions tend to have enormous symptom burden, variable disease trajectory, and poor prognoses that affect patients as well as their families and caregivers. ${ }^{2,3}$ General as well as specialized palliative care is required in neurologically injured patients, such as those with Parkinson disease (PD), dementia, amyotrophic lateral sclerosis, brain tumors, stroke, and acute neurologic illnesses to address their complex needs. ${ }^{3-5}$ The comprehensive approach includes care of physical, psychological, social, and spiritual aspects of patients to provide comfort and to improve their quality of life. Palliative care also assesses and treats other sources of distress such as normal reactions to living with a life-threatening, progressive, and/or disabling illness. In neurological and neurosurgical patients, the concept of autonomy in clinical practice can only be achieved by adopting the principles of palliative care. With declining cognitive or physical function, the discussion about prognosis, goals of care, and advance care planning will improve the patient outcome and caregiver satisfaction.

Neurological diseases are largely incurable due to multiple factors and considerably reduce the quality of life due to associated pain, anxiety, depression and other symptoms that are difficult to control. Hence, early integration of palliative care in neurological patients will improve the overall well-being of patients and their family members. ${ }^{6-8}$ Miyasaki et al showed that symptom burden on patients and caregivers in advanced (PD) is similar to that in advanced malignancy. ${ }^{9}$ Hence, there is growing need of integration of palliative care approach in neurology practice. ${ }^{1,10}$ The palliative approach in traditional neurology benefits patients in many ways. As traditional approaches concentrate more on the preservation of function and prolongation of life, palliative care emphasizes more on symptom management and relief from suffering. It also prepares the patient and family

Address for correspondence Sachidanand J. Bharati, MD, DM, Onco-Anaesthesia and Palliative Medicine, Dr. BRA Institute Rotary Cancer Hospital, All India Institute of Medical Sciences (AlIMS), Ansari Nagar, New Delhi-110029, India (e-mail: sachidadr@yahoo.co.in). members to accept death as natural outcome rather than as a failure of medical treatment. ${ }^{3,4}$ Neurologists have a primary responsibility to assess and treat nonmotor symptoms, such as pain, depression, anxiety, fatigue, sleep, constipation, urinary urgency, and sexual dysfunction. Studies in different type of neurological populations have shown that nonmotor symptoms are the most debilitating for patients, and these symptoms affect caregiver burden and overall quality of life more than motor symptoms. ${ }^{11-13}$ Most common neurological and neurosurgical diseases that require palliative care are chronic neurodegenerative disorders, motor neuron disease, ischemic or hemorrhagic stroke, demyelinating diseases, movement disorders, and brain tumors.

This is high time to incorporate fundamental palliative care skills, including communicating bad news, nonmotor symptom assessment and management, advance care planning, and caregiver assessment in neurology practice and specialist palliative care referral for more complex or advanced patients. The modes of specialist palliative care services can be inpatient palliative care consultation, outpatient palliative care clinics, home palliative care, or hospice. But traditional models of palliative care may not sufficiently address the specific needs of patients and family members living with a neurological diagnosis. Patients with a life-limiting neurological illness have a different disease trajectory as compared to cancer patients. Apart from physical symptoms, other predominant problems include cognitive impairment, behavioral issues, and communication difficulties. ${ }^{14,15}$ The notable differences between neurology and other patients include symptom profiles, psychosocial issues, caregiver needs, and effects on spiritual well-being. The patients with motor neuron disease have more demoralization, hopelessness, and suicidal ideation than patients with metastatic cancer. ${ }^{16}$ Similarly, patients with brain tumors have distinct issues, including cognitive problems, seizures, and communication difficulties, than patients living with other types of cancers. ${ }^{17}$; Patients with Huntington

Copyright $\odot 2019$ Indian Society of Neuroanaesthesiology and Critical
Care

\section{License terms}

$(\circledast) \Theta \circledast$
DOI https://doi.org/

10.1055/s-0039-1679195 ISSN 2348-0548. 
disease have special social needs as a result of combined behavioral, psychiatric, movement, and cognitive issues. ${ }^{18}$ Palliative care needs of patients with malignant gliomas are also quite different from others in the cancer patient population. This is because of different trajectory of disease, short life expectancy, and the presence of specific symptoms related to neurological deterioration. The specific issues include management of seizures, peritumoral edema, venous thromboembolism, depression, and opportunistic infections. The other problems include psychological issues, communication problems, need for rehabilitation, and decision on end-of-life treatments and choices. ${ }^{19-22}$

Specialist palliative care referral may be warranted in end-of-life care, feeding tube discussions or other complex interventions, spiritual issues arising from prolonged illness, distressing psychological issues, and in intractable physical symptoms. Also, frequent hospital admissions due to pneumonia, falls, and urinary tract infection, ongoing weight loss, progressive dysphagia, restricted activities of daily living, or a rapid decline in function may signify the need for referral to hospice. ${ }^{4}$

Neuropalliative care is developing as a subspecialty but there are several questions that need to be answered including:

1. Development and validation of prognostic predictors.

2. Tools for identification of high-risk patients to be referred to specialist palliative care services.

3. Developing evidences for better control of nonmotor symptoms.

4. Cost-effectiveness of palliative care interventions and the model for palliative care delivery.

To conclude, though the patients with neurological disorders frequently have very complex needs, these are still manageable using a palliative approach. Early communication with patients and family members about various issues, such as disease trajectory, common symptoms, treatment options, and prognosis, may be helpful in alleviating distress experienced by them. This timely communication helps in building a strong clinician-patient relationship that forms the basis of good care. Further, to ensure that the care provided by clinician aligns with the patient preferences, shared decision making regarding critical decisions throughout the continuum of disease is essential. However, more research is needed to determine the ideal method of incorporating palliative care into the management plans for patients with a variety of neurological conditions.

\section{Conflict of Interest}

None declared.

\section{References}

1 The American Academy of Neurology Ethics and Humanities Subcommittee. Palliative care in neurology. Neurology 1996;46(3):870-872

2 Hudson PL, Aranda S, Kristjanson LJ. Meeting the supportive needs of family caregivers in palliative care: challenges for health professionals. J Palliat Med 2004;7(1):19-25
3 Lanoix M. Palliative care and Parkinson's disease: managing the chronic-palliative interface. Chronic Illn 2009;5(1):46-55

4 Chahine LM, Malik B, Davis M. Palliative care needs of patients with neurologic or neurosurgical conditions. Eur J Neurol 2008;15(12):1265-1272

5 Bindu B, Rath GP. Palliative Care for Neurologically Injured Patients: Why and How? Journal of Neuroanaesthesiology and Critical Care 2019;6(1):5-12

6 Hoyert DL, Xu J. Deaths: Preliminary Data for 2011. Natl Vital Stat Rep 2012;61(6):1-51

7 Martinez-Martin P, Schapira AH, Stocchi F, et al. Prevalence of nonmotor symptoms in Parkinson's disease in an international setting; study using nonmotor symptoms questionnaire in 545 patients. Mov Disord 2007;22(11):1623-1629

8 Kim Y, Schulz R. Family caregivers' strains: comparative analysis of cancer caregiving with dementia, diabetes, and frail elderly caregiving. J Aging Health 2008;20(5):483-503

9 Miyasaki JM, Long J, Mancini D, et al. Palliative care for advanced Parkinson disease: an interdisciplinary clinic and new scale, the ESAS-PD. Parkinsonism Relat Disord 2012;18(Suppl 3):S6-S9

10 Foley KM, Carver AC. Palliative care in neurology. Neurol Clin 2001;19(4):789-799

11 Cheon SM, Ha MS, Park MJ, Kim JW. Nonmotor symptoms of Parkinson's disease: prevalence and awareness of patients and families. Parkinsonism Relat Disord 2008;14(4):286-290

12 Fruehwald S, Loeffler-Stastka H, Eher R, Saletu B, Baumhackl $\mathrm{U}$. Depression and quality of life in multiple sclerosis. Acta Neurol Scand 2001;104(5):257-261

13 Simmons Z, Bremer BA, Robbins RA, Walsh SM, Fischer S. Quality of life in ALS depends on factors other than strength and physical function. Neurology 2000;55(3):388-392

14 Turner-Stokes L, Sykes N, Silber E, Khatri A, Sutton L, Young E. From diagnosis to death: exploring the interface between neurology, rehabilitation and palliative care in managing people with long-term neurological conditions. Clin Med (Lond) 2007;7(2):129-136

15 Teno JM, Weitzen S, Fennell ML, Mor V. Dying trajectory in the last year of life: does cancer trajectory fit other diseases? J Palliat Med 2001;4(4):457-464

16 Clarke DM, McLeod JE, Smith GC, Trauer T, Kissane DW. A comparison of psychosocial and physical functioning in patients with motor neurone disease and metastatic cancer. J Palliat Care 2005;21(3):173-179

17 Schubart JR, Kinzie MB, Farace E. Caring for the brain tumor patient: family caregiver burden and unmet needs. Neuro Oncol 2008;10(1):61-72

18 Phillips W, Shannon KM, Barker RA. The current clinical management of Huntington's disease. Mov Disord 2008;23 (11):1491-1504

19 Catt S, Chalmers A, Fallowfield L. Psychosocial and supportive-care needs in high-grade glioma. Lancet Oncol 2008;9(9):884-891

20 Finocchiaro CY, Petruzzi A, Lamperti E, et al. The burden of brain tumor: a single-institution study on psychological patterns in caregivers. J Neuro Oncol 2012;107(1):175-181

21 Flechl B, Ackerl M, Sax C, et al. The caregivers' perspective on the end-of-life phase of glioblastoma patients. J Neuro Oncol 2013;112(3):403-411

22 Ford E, Catt S, Chalmers A, Fallowfield L. Systematic review of supportive care needs in patients with primary malignant brain tumors. Neuro Oncol 2012;14(4):392-404 